\title{
Cap myopathy
}

INSERM

\section{Source}

INSERM. (1999). Orphanet: an online rare disease and orphan drug data base. Cap

myopathy. ORPHA:171881

Cap myopathy is a very rare congenital myopathy presenting a weakness of facial and respiratory muscles associated with craniofacial and thoracic deformities, as well as weakness of limb proximal and distal muscles. Onset is at birth or in childhood, weakness progression is slow but may lead to a severe and even fatal prognosis. 